\title{
ADUBAÇÃO COM NITROGÊNIO E BORO NA INCIDÊNCIA DE HASTES OCAS E NA PRODUÇÃO DE COUVE-FLOR
}

\author{
Nitrogen and boron fertilization on hollow stem and yield of cauliflower
}

\author{
Cíntia Kojoi ${ }^{1}$, Simone da Costa Mello², Mônica Sartori de Camargo ${ }^{3}$, Evandro Binotto Fagan ${ }^{4}$, Maurício Ferreira Ribeiro ${ }^{5}$
}

\section{RESUMO}

O aparecimento de caule oco em couve-flor causa prejuízos aos produtores. O boro e o nitrogênio estão associados a essa desordem fisiológica, mas poucos estudos foram feitos em condições tropicais. Objetivou-se, no trabalho, avaliar os efeitos da adubação com N e B e da interação entre esses nutrientes na produção e na ocorrência de hastes ocas em couve-flor 'Shiromaru III', cultivada em solo da região de Ribeirão Preto (SP), durante o período de julho a setembro de 2003. O experimento foi conduzido em blocos inteiramente casualizados em esquema fatorial $(3 \mathrm{x} 3)$, com três doses de $\mathrm{N}\left(120,180 \mathrm{e} 240 \mathrm{~kg} \mathrm{ha}^{-1}\right)$ e três doses de $\mathrm{B}(2,4 \mathrm{e}$ $6 \mathrm{~kg} \mathrm{ha}^{-1}$ ) e 4 repetições. A produção total e a massa média de inflorescências não foram influenciadas pelas doses de $\mathrm{N}$ e de B. A incidência de hastes ocas foi influenciada pela adubação com N, sendo que as aplicações de 180 e $240 \mathrm{~kg} \mathrm{ha}^{-1}$ reduziram o aparecimento dessa anomalia em $9,03 \%$ e $11,72 \%$, respectivamente.

Termos para indexação: Brassica oleracea, solo, desordem nutricional.

\section{ABSTRACT}

The hollow stem in cauliflower causes damages to producers. Nitrogen and boron are related to this physiological disorder but few studies were performed at tropical conditions. The aim of the experiment was to evaluate the effects of $\mathrm{N}$ and $\mathrm{B}$ applications and interaction between these nutrients on yield and hollow stem on cauliflower Shiromaru III, cultivated on soil of Ribeirão Preto region during the period from July to September of 2003. The experiment was carried out in a completely randomized design in a factorial scheme ( $3 \times 3)$ with three rates of nitrogen $\left(120,180\right.$ and $\left.240 \mathrm{~kg} \mathrm{ha}^{-1} \mathrm{~N}\right)$ and three rates of boron $\left(2,4\right.$ and $\left.6 \mathrm{~kg} \mathrm{ha}{ }^{-1} \mathrm{~B}\right)$ and four replicates. The total yield and head weight were not influenced by B and $\mathrm{N}$ applications. Hollow stem incidence was influenced by $\mathrm{N}$ fertilization, where applications of 180 and $240 \mathrm{~kg} \mathrm{ha}^{-1}$ reduced the disorder in 9.03 and $11.72 \%$, respectively.

Index terms: Brassica oleracea, soil, nutritional disorder.

(Recebido em 23 de outubro de 2007 e aprovado em 3 de junho de 2008)

\section{INTRODUÇÃo}

O boro (B) é importante para a produção de hortaliças, pois sua deficiência causa mudanças fisiológicas, bioquímicas e anatômicas nas plantas, incluindo alterações na estrutura da parede celular, no funcionamento e na integridade das membranas, nas atividades enzimáticas e na produção de compostos metabólitos (GOLDBACK, 1997).

Em couve-flor (Brassica oleracea var. botrytis ), os sintomas visuais de deficiência de B são conhecidos pelo aparecimento de pontuações escuras na inflorescência e cavidade na parte interna da haste. Essas desordens estão relacionadas à distribuição e mobilidade do $\mathrm{B}$ na planta e por fatores que afetam esses mecanismos e a disponibilidade do elemento no solo. O transporte do B é realizado via xilema, cuja distribuição depende da perda de água por transpiração pelos órgãos vegetais. Como os sítios de maior transpiração são as folhas desenvolvidas, o fluxo de B é mais intenso para esses tecidos que não são os de maior demanda por B, como as inflorescências. O B, dependendo da espécie, é considerado um elemento imóvel no floema, ou seja, praticamente não ocorre sua redistribuição das folhas desenvolvidas para as inflorescências e outros órgãos de crescimento que transpiram menos (SHELP et al., 1995).

Os fatores que podem afetar a disponibilidade de B no solo e os mecanismos de absorção e distribuição deste elemento na planta incluem as características físicas e químicas do solo, interação do B com outros nutrientes,

\footnotetext{
'Engenheira Agrônoma - Faculdade de Ciências Agráricas/FACIAGRA - Centro Universitário de Patos de Minas - Rua Augusto da Silva Barão - 38700 -090 Patos de Minas, MG

"Engenheira Agrônoma, Doutora, Professora - Departamento de Produção Vegetal/LPV - Escola Superior de Agricultura "Luiz de Queiroz"/ESALQ Universidade de São Paulo/USP - Avenida Pádua Dias, 11 - Cx. P. 9 - 13418-900 - Piracicaba, SP - scmello@esalq.usp.br

${ }^{3}$ Engenheira Agrônoma, Doutora, Pesquisadora - APTA Regional Centro Sul - Rodovia SP 127, Km 30 - Vila Fátima - Cx. P. 28 - $13400-970$ - Piracicaba, SP mscamarg@yahoo.com.br

${ }^{4}$ Engenheiro Agrônomo, Doutor - Faculdade de Ciências Agráricas/FACIAGRA - Centro Universitário de Patos de Minas - Rua Augusto da Silva Barão 38700-090 - Patos de Minas, MG - evbinotto@yahoo.com.br

${ }^{5}$ Engenheiro Agrônomo - Instituição Moura Lacerda - Rua Padre Euclides, 995 - 14085-420 - Ribeirão Preto, SP
} 
dose e método de aplicação desse elemento, condições climáticas e características genéticas das cultivares (GUPTA, 1993).

A interação do B com outros nutrientes, principalmente com $\mathrm{N}$, tem sido muito investigada, pois o $\mathrm{N}$ é um dos elementos que mais afetam a absorção desse micronutriente e o crescimento da couve-flor. Segundo Shelp et al. (1995), a absorção de B não acompanha a velocidade de crescimento de plantas de couve-flor, quando adubadas com elevadas quantidades de $\mathrm{N}$ no solo, induzindo o aparecimento de hastes ocas. Elevadas concentrações de $\mathrm{N}$ nítrico ou amoniacal também diminuem os teores de B nas plantas pelo provável antagonismo dessas formas nitrogenadas com o B.

Entretanto, o efeito da interação entre $\mathrm{N}$ e B sobre o aparecimento de hastes ocas em couve-flor tem sido contraditório. Para Everaarts et al. (2000), a aplicação de 2 $\mathrm{kg} \mathrm{ha}^{-1}$ de B não influenciou a ocorrência de cavidade na haste e a adubação nitrogenada resultou no aparecimento dessa desordem fisiológica, por ter proporcionado crescimento mais acelerado da planta. Em dois locais, Batal et al. (1997) não obtiveram redução na incidência de haste oca com o aumento das doses de B $\left(2,2\right.$ a $\left.8,8 \mathrm{~kg} \mathrm{ha}^{-1}\right)$ quando associadas com alta taxa de $\mathrm{N}\left(269 \mathrm{~kg} \mathrm{ha}^{-1}\right)$, também pelo fato dessa quantidade de $\mathrm{N}$ ter influenciado o desenvolvimento da planta, o que foi confirmado pelas correlações negativas entre a massa da inflorescência e a porcentagem de plantas com hastes ocas $(\mathrm{r}=-0,321, \mathrm{P}<$ 0,001 e $r=-0,466, P<0,001$, em cultivares de couve-flor Tifton e Plains, respectivamente). Por outro lado, Kotur (1997), cultivando couve-flor em solo deficiente em B (0,1 $\mathrm{mg} \mathrm{kg}^{-1}$ ), obteve redução no número de plantas com hastes ocas através da adubação com $\mathrm{B}$, que foi mais expressiva quando esse micronutriente foi associado com dose elevada de $\mathrm{N}$ (300 $\left.\mathrm{kg} \mathrm{ha}^{-1}\right)$.

Dessa forma, objetivou-se, no trabalho, avaliar a influência da adubação com N e B na produção e na incidência de haste oca em couve-flor 'Shiromaru III', na região de Ribeirão Preto $(\mathrm{SP})$.

\section{MATERIALE MÉTODOS}

$\mathrm{O}$ experimento foi conduzido na área experimental da Instituição Moura Lacerda, Ribeirão Preto (SP), durante o período de julho a setembro de 2003. Foi utilizado o híbrido Shiromaru III em função da boa produtividade, aceitação no mercado local e suscetibilidade à deficiência de boro.

O solo da área experimental, um Latossolo Vermelho textura argilosa, apresentou os seguintes resultados quanto à análise química: matéria orgânica $=28 \mathrm{~g} \mathrm{~kg}^{-1}$ (dicromato de potássio); $\mathrm{P}$ (resina) = $11 \mathrm{mg} \mathrm{dm}^{-3} ; \mathrm{K}, \mathrm{Ca}$ e $\mathrm{Mg}$ (resina) $=1,3$; 8,0 e 4,0 mmol dm $\mathrm{dm}^{-3} ; \mathrm{pH}\left(\mathrm{CaCl}_{2}\right)=5,9 ; \mathrm{S}=84,7 \mathrm{mg} \mathrm{dm}^{-3} ; \mathrm{CTC}$ $=67 \mathrm{mmol} \mathrm{dm}^{-3} ; \mathrm{V} \%=20 ; \mathrm{B}$ (água quente) $=0,35 \mathrm{mg} \mathrm{dm}^{-3} . \mathrm{Em}$ função da análise química do solo aplicou-se o equivalente a $4 \mathrm{t} \mathrm{ha}^{-1}$ de calcário dolomítico, com o objetivo de elevar a saturação por bases a $80 \%$ e o teor de $\mathrm{Mg}$ a $9 \mathrm{mmol}_{\mathrm{c}} \mathrm{dm}^{-3}$. Após o preparo do solo, realizou-se a adubação de plantio de acordo com as recomendações de Trani et al. (1996), empregando-se $60 \mathrm{~kg} \mathrm{ha}^{-1} \mathrm{de} \mathrm{N}, 600 \mathrm{~kg} \mathrm{ha}^{-1} \mathrm{de}_{2} \mathrm{P}_{5}$ e $240 \mathrm{~kg}$ $\mathrm{ha}^{-1}$ de $\mathrm{K}_{2} \mathrm{O}$, na forma de uréia $(45 \% \mathrm{~N})$, de superfosfato simples $\left(18 \% \mathrm{P}_{2} \mathrm{O}_{5}\right)$ e de cloreto de potássio $\left(60 \% \mathrm{~K}_{2} \mathrm{O}\right)$.

$\mathrm{O}$ experimento foi instalado em blocos casualizados com quatro repetições, sendo os tratamentos compostos por três doses de $\mathrm{N}\left(120,180\right.$ e $\left.240 \mathrm{~kg} \mathrm{ha}^{-1}\right)$ e três doses de B (2, 4 e $\left.6 \mathrm{~kg} \mathrm{ha}^{-1}\right)$, aplicadas no solo. O boro, na forma de bórax $(11,5 \%)$, foi aplicado no transplante das mudas e no início da formação das inflorescências de couve-flor, em duas partes iguais que foram dissolvidas em água, utilizando $0,5 \mathrm{~L}$ de solução por planta. As doses de $\mathrm{N}$ foram divididas em três partes iguais e aplicadas aos 15, 30 e 45 dias após o transplante. Aos 30 e 45 dias, as plantas também foram pulverizadas com uma solução contendo molibdato de amônio a $0,1 \%$ e cloreto de cálcio a $0,6 \%$.

Plântulas de couve-flor 'Shiromaru III' foram produzidas em bandejas de isopor contendo 200 células preenchidas com substrato orgânico à base de casca de pinus, em condições de casa-de-vegetação. $O$ transplante das mudas, em sulcos, ocorreu aos 35 dias após a semeadura (01/07/2003), no espaçamento de $1 \mathrm{~m}$ entre linhas e 0,5 m entre plantas. Cada parcela foi composta de 20 plantas distribuídas em duas linhas de plantio. As parcelas apresentavam $1 \mathrm{~m}$ de distância entre si para evitar contaminação entre os tratamentos.

Aos 56 dias após o transplante, foram coletadas cinco folhas por parcela, uma folha de cada planta, retirando-se a folha desenvolvida no período da formação da inflorescência. As folhas foram lavadas e secadas em estufa a $65^{\circ} \mathrm{C}$ por 48 horas. Posteriormente, procedeu-se a moagem do material vegetal para a realização da análise química de macronutrientes e de B, segundo metodologia descrita em Malavolta et al. (1997).

A colheita das inflorescências iniciou-se aos 71 dias após o transplante das mudas, estendendo-se por 17 dias. Duas plantas de cada extremidade da fileira na parcela foram deixadas como bordadura e as inflorescências das demais foram colhidas, e avaliadas quanto à presença de cavidade na haste. Além disso, foram determinadas a produção total e a produção comercial.

Os dados foram submetidos à análise de variância pelo teste $\mathrm{F}$, e a comparação entre médias pelo teste Tukey, ao nível de $5 \%$ de probabilidade. 


\section{RESULTADOS E DISCUSSÃO}

Para a porcentagem de plantas com hastes ocas, não foi constatada interação significativa entre as doses de $\mathrm{N}$ e de $\mathrm{B}$. A incidência dessa anomalia foi influenciada somente pelas doses de $\mathrm{N}$, sendo que as aplicações de 180 e $240 \mathrm{~kg} \mathrm{ha}^{-1}$ reduziram o aparecimento de hastes ocas para 12,60 e 9,91\%, respectivamente, em relação à menor dose, cuja incidência foi elevada $(21,63 \%)$.

Resultados semelhantes foram obtidos por Scaife \& Wurr (1990) através da diminuição na severidade de hastes ocas em couve-flor com a aplicação de $225 \mathrm{~kg} \mathrm{ha}^{-1}$ de N. Para Kotur (1997) também foi possível observar redução no grau de severidade dessa anomalia, que passou de 3,7 para 2,8 (escala de notas de 1 a 5) em plantas que receberam 50 e $150 \mathrm{~kg} \mathrm{ha}^{-1}$ de $\mathrm{N}$, respectivamente. Para outros autores (BÉLEC et al., 2001; EVERAARTS et al., 2000; VIGIER \& CUTCLIFFE, 1984), no entanto, doses elevadas de $\mathrm{N}$ aumentaram a incidência de hastes ocas em couve-flor por acelerarem a taxa de crescimento da planta, o que contribuiu para a diluição da concentração de B. A explicação para esses resultados discordantes se baseia na influência das características genéticas sobre o crescimento e a maturação da planta (BATAL et al., 1997).

As doses de B não influenciaram a ocorrência de hastes ocas, corroborando com os resultados obtidos por Vigier \& Cutcliffe (1984) em plantas de brócolis, cujas aplicações de 4 e de $8 \mathrm{~kg} \mathrm{ha}^{-1}$ de $\mathrm{B}$ não afetaram o aparecimento dessa desordem fisiológica. Para Gupta \& Cutclife (1973), o B sozinho pode não ser capaz de reduzir a incidência de hastes ocas, pois o efeito da sua aplicação depende de outros fatores como das condições climáticas, das práticas de manejo e das características da cultivar. Quanto às condições climáticas, o período em que foi realizado o experimento não foi caracterizado por elevadas temperaturas e precipitações, o que poderia tornar o processo de crescimento mais rápido que a absorção de $\mathrm{B}$, resultando no aparecimento de hastes ocas. Para as características das cultivares, Shelp et al. (1995) mostraram diferenças entre as variedades de couve-flor quanto à absorção de boro, conseqüientemente ao aparecimento de hastes ocas.

As quantidades elevadas de B não provocaram sintomas de toxicidade nas plantas em início de desenvolvimento, possivelmente pelo método usado, que consistiu na aplicação parcelada de bórax $(11 \% \mathrm{~B})$ dissolvido em água, no transplante das mudas e no início da formação da inflorescência da couve-flor. Outros métodos de aplicação, porém, em faixas ao longo da linha de plantio, na dosagem de 4 e $8 \mathrm{~kg} \mathrm{ha}^{-1}$ de B (VIGIER \& CUTCLIFFE, 1984), ou em covas, com 1,6 kg ha' ${ }^{-1}$ de B (MELLO et al., 1997), induziram sintomas de toxicidade desse elemento em plantas de brócolis.

Houve efeito da aplicação de $\mathrm{N}$, dentro de cada dose de B, para a produção comercial de couve-flor. Nas áreas adubadas com 2 e $4 \mathrm{~kg} \mathrm{ha}^{-1} \mathrm{de} \mathrm{B}$, as maiores produções comerciais foram obtidas com $180 \mathrm{~kg} \mathrm{ha}^{-1}$ de $\mathrm{N}$ em relação à maior dose. Entretanto, a produção referente à aplicação de $180 \mathrm{~kg} \mathrm{ha}^{-1}$ de $\mathrm{N}$ não diferiu significativamente daquela obtida com a menor dose. Com a adubação de $6 \mathrm{~kg} \mathrm{ha}^{-1} \mathrm{de}$ B, os níveis de nitrogênio não influenciaram a produção comercial de couve-flor (Tabela 1). Interação significativa também foi observada por Kotur (1997) para a produção de couve-flor, cujo valor máximo foi obtido com a aplicação de $200 \mathrm{~kg} \mathrm{ha}^{-1}$ de $\mathrm{N}$ na taxa de $0,125 \%$ de B. Batal et al. (1997), por sua vez, não observaram interação entre N e B sobre a produção de couve-flor. Esses resultados confirmam que a interação entre esses elementos é complexa.

A produção total e a massa média de inflorescências de couve-flor não foram afetadas com as aplicações de $\mathrm{N}$ e de B, possivelmente pelo tipo de solo e pela sua fertilidade (Tabela 2). Da mesma forma, Everaarts \& Moel (1995) não constataram aumento no tamanho das inflorescências de couve-flor com a aplicação de N, em solo com quantidade elevada de N mineral. Em Batal et al. (1997), a produção total e a massa média de inflorescências de couve-flor não foram influenciadas pelas aplicações de 2,2; 4,4 e 8,8 $\mathrm{kg} \mathrm{ha}^{-1}$ de B, em solo com $0,21 \mathrm{mg} \mathrm{kg}^{-1}$ de B e alta CTC. Já em solo com baixo teor de B $\left(0,07 \mathrm{mg} \mathrm{dm}^{-3}\right)$ e CTC de 25,5 $\mathrm{mmol} \mathrm{dm}^{-3}$, os autores obtiveram aumento nos valores dessas características com a aplicação de $4,4 \mathrm{~kg} \mathrm{ha}^{-1}$ de B, mostrando a influência da fertilidade do solo na resposta da planta à adição de boro. Quanto ao N, cabe ressaltar, ainda, que o parcelamento da adubação em três períodos pode ter contribuído para o efeito não significativo desse nutriente na produção de couve-flor (BABIK et al., 1994; BRACY et al., 1995).

$A$ adição de $B$ aumentou o teor foliar desse elemento (Tabela 3), concordando com os resultados obtidos por Gupta \& Cutcliffe (1973, 1975), Shelp et al. (1992) e Vigier \& Cutcliffe (1984). Os teores foliares de potássio e enxofre foram reduzidos com as doses de B. Entretanto, eles permaneceram dentro do intervalo considerado adequado para cada nutriente (TRANI \& RAIJ, 1996). Os teores de N, P, Ca e Mg nas folhas, por sua vez, não sofreram influência da adubação com B e N, cujos valores médios encontrados foram respectivamente: 61,$5 ; 7,9 ; 20,2$ e $3,5 \mathrm{~g} \mathrm{~kg}^{-1}$. 
Tabela 1 - Efeito da aplicação de N, em cada dose de B, na produção comercial (t ha $\left.{ }^{-1}\right)$ de couve-flor 'Shiromaru III'. Ribeirão Preto-SP, 2003.

\begin{tabular}{|c|c|c|c|}
\hline \multirow[t]{2}{*}{ Boro $\left(\mathrm{kg} \mathrm{ha}^{-1}\right)$} & \multicolumn{3}{|c|}{ Nitrogênio $\left(\mathrm{kg} \mathrm{ha}^{-1}\right)$} \\
\hline & 120 & 180 & 240 \\
\hline 2 & $29,07 \mathrm{AB}$ & $32,82 \mathrm{~A}$ & $28,10 \mathrm{~B}$ \\
\hline 4 & $32,10 \mathrm{AB}$ & $34,27 \mathrm{~A}$ & $29,65 \mathrm{~B}$ \\
\hline 6 & $32,67 \mathrm{~A}$ & $30,02 \mathrm{~A}$ & $32,37 \mathrm{~A}$ \\
\hline Teste F & & & $3,19 * *$ \\
\hline C.V. $(\%)$ & & & 7,85 \\
\hline DMS & & & 4,33 \\
\hline
\end{tabular}

** Significativo $(\mathrm{P}<0,01)$

Médias seguidas pela mesma letra na linha não diferem entre si pelo teste Tukey, a 5\% de probabilidade.

Tabela 2 - Produção total (PT) e massa média das inflorescências (M) de couve-flor 'Shiromaru III', em função das aplicações de nitrogênio e boro. Ribeirão Preto-SP, 2003.

\begin{tabular}{|c|c|c|c|c|c|c|}
\hline \multirow[t]{3}{*}{ Boro $\left(\mathrm{kg} \mathrm{ha}^{-1}\right)$} & \multicolumn{6}{|c|}{ Nitrogênio $\left(\mathrm{kg} \mathrm{ha}^{-1}\right)$} \\
\hline & \multicolumn{2}{|c|}{120} & \multicolumn{2}{|c|}{180} & \multicolumn{2}{|c|}{240} \\
\hline & $\mathrm{PT}\left(\mathrm{t} \mathrm{ha}^{-1}\right)$ & $M(g)$ & PT $\left(\mathrm{t} \mathrm{ha}^{-1}\right)$ & M (g) & PT $\left(\mathrm{t} \mathrm{ha}^{-1}\right)$ & $M(g)$ \\
\hline 2 & 33,6 & 1667,7 & 37,2 & 1859,1 & 33,3 & 1664,8 \\
\hline 4 & 34,0 & 1702,6 & 35,4 & 1770,1 & 32,2 & 1610,9 \\
\hline \multirow[t]{2}{*}{6} & 32,7 & 1633,8 & 32,3 & 1613,5 & 33,3 & 1655,4 \\
\hline & PT $\left(\mathrm{t} \mathrm{ha}^{-1}\right)$ & $M(g)$ & & & & \\
\hline Teste F & $1,03 \mathrm{NS}$ & $1,81 \mathrm{NS}$ & & & & \\
\hline C.V. $(\%)$ & 8,39 & 10,31 & & & & \\
\hline DMS & 2,89 & 175,00 & & & & \\
\hline
\end{tabular}

NS = Não significativo a $5 \%$ de probabilidade.

Tabela 3 - Teores foliares de potássio (K), de enxofre (S) e de boro (B) em couve-flor 'Shiromaru III', em função das doses de boro. Ribeirão Preto-SP, 2003.

\begin{tabular}{cccc}
\hline Boro $\left(\mathrm{kg} \mathrm{ha}^{-1}\right)$ & $\mathrm{K}\left(\mathrm{g} \mathrm{kg}^{-1}\right)$ & $\mathrm{S}\left(\mathrm{g} \mathrm{kg}^{-1}\right)$ & $\mathrm{B}\left(\mathrm{mg} \mathrm{kg}^{-1}\right)$ \\
\hline 2 & $32,29 \mathrm{a}$ & $9,02 \mathrm{ab}$ & $18,39 \mathrm{c}$ \\
4 & $30,83 \mathrm{ab}$ & $9,33 \mathrm{a}$ & $26,97 \mathrm{~b}$ \\
6 & $29,61 \mathrm{~b}$ & $8,77 \mathrm{~b}$ & $33,34 \mathrm{a}$ \\
\hline Teste F & $3,60 *$ & $7,32 * *$ & $43,08 * *$ \\
C.V. $(\%)$ & 7,90 & 4,00 & 15,09 \\
\hline DMS & 2,49 & 0,37 & 4,03 \\
\hline
\end{tabular}

* e ** Significativo a $5 \%$ e $1 \%$ de probabilidade, respectivamente.

Médias seguidas pela mesma letra na coluna não diferem entre si pelo teste Tukey, a 5\% de probabilidade.

\section{CONCLUSÕES}

A incidência de hastes ocas em couve-flor 'Shiromaru III' foi reduzida com as aplicações de 180 e $240 \mathrm{~kg} \mathrm{ha}^{-1} \mathrm{~N}$ em solo argiloso, com teor médio de $\mathrm{B}\left(0,35 \mathrm{mg} \mathrm{kg}^{-1}\right)$.
A produção total e a massa média das inflorescências não foram influenciadas pelas doses de $\mathrm{N}$ e de $\mathrm{B}$.

A produção comercial foi superior com a aplicação de $180 \mathrm{~kg} \mathrm{ha}^{-1}$ de $\mathrm{N}$ em relação à maior dose, na presença de 2 ou $4 \mathrm{~kg} \mathrm{ha}^{-1}$ de B. 
$\mathrm{O} \mathrm{N}$ influencia o aparecimento de hastes ocas, independente da adubação com boro.

\section{REFERÊNCIAS BIBLIOGRÁFICAS}

BABIK, I.; RUMPEL, J.; ELKNER, K. The influence of nitrogen fertilization on yield, quality a senescence of Brussels sprouts. Acta Horticulturae, The Hague, v. 404, p. 353-359, 1994.

BATAL, K. M.; GRANBERRY, D. M.; MULLINIX JUNIOR, B. G. Nitrogen, magnesium, and boron applications affect cauliflower yield, curd mass, and hollow stem disorder. Hortscience, Saint Joseph, v. 32, n. 1, p. 75-78, 1997.

BÉLEC, C.; VILLENEUVE, S.; COULOMBE, J.; TREMBLAY, $\mathrm{N}$. Influence of nitrogen fertilization on yield, hollow stem incidence and sap nitrate concentration in broccoli. Canadian Journal of Plant Science, Ottawa, v. 81, p. 765$772,2001$.

BRACY, P. R.; PARISH, R. L.; BERGERON, P. E. Sidress N application methods for broccoli production. Journal of Vegetable Crop Production, Louisiana, v. 1, p. 63-71, 1995.

EVERAARTS, A. P.; MOEL, C. P. de. The effect of nitrogen and the method of application on the yield of cauliflower. Netherlands Journal of Agricultural Science, Wageningen, v. 43, p. 409-418, 1995.

EVERAARTS, A. P.; PUTTER, H. de; PUTTER, H. de. Fast growth results in more hollow stems in cauliflower.PVA Bulletin Vollegrondsroenteteelt, Netherlands, v. 2, p. 4-6, 2000.

GOLDBACK, H. E. A critical review on current hypotheses concerning the role of boron in higher plants: suggestions for further research and methodological requirements. Journal Trace Microprobe Techniques, v. 15, p. 51-91, 1997.

GUPTA, U. C. Boron deficiency and toxicity symptoms for several crops as related to tissue boron levels. Journal Plant Nutrition, New York, v. 6, n. 5, p. 387-395, 1983.

GUPTA, U. C.; CUTCLIFFE, J. A. Boron nutrition of broccoli, Brussels sprouts and cauliflower grown on Prince Edward Island soils. Canadian Journal of Soil Science, Ottawa, v. 53, p. 275-279, 1973.

GUPTA, V. C.; CUTCLIFFE, J. A. Boron deficiency in cole crops under field and greenhouse conditions.
Communications in Soil Science and Plant Analysis, New York, v. 6, p. 181-188, 1975.

KOTUR, S. C. Nitrogen-boron interaction in cauliflower (Brassica oleraceae var. botrytis L.) on an Alfisol. Journal of the Indian Society of Soil Science, New Delhi, v. 45, n. 3, p. 519-522, 1997.

MALAVOLTA, E.; VITTI, G. C.; OLIVEIRA, S. A. Avaliação do estado nutricional das plantas: princípios e aplicações. Piracicaba: Potafos, 1997. 201 p.

MELLO, S. C.; CASTELlANE, P. D.; CORTEZ, G. E. P. Influência do boro no desenvolvimento e na produtividade de cultivares de brócoli (Brassica oleracea var. itálica). Científica, Jaboticabal, v. 25, n. 2, p. 269277, 1997.

SCAIFE, A.; WURR, D. C. E. Effects of nitrogen and irrigation on hollow stem of cauliflower (Brassica oleraceae var botrytis). Journal of Horticultural Science, London, v. 65 , n. 1 , p. 25-29, 1990.

SHELP, B. J.; MARENTES, E.; KITHEKA, A. M.; VIVEKANANDAN, P. Boron mobility in plants. Physiologia Plantarum, Kobenhavn, v. 94, p. 356-361, 1995.

SHELP, B. J.; PENNER, R.; ZHU, Z. Broccoli (Brassica oleracea var.italica) cultivar response to boron deficiency. Canadian Journal Plant Science, Otawa, v. 72, p. 883-888, 1992.

TRANI, P. E.; NAGAI, H.; PASSOS, F. A. Brócolos, couveflor e repolho. In: RAIJ, B. van; CANTARELLA, H.; QUAGgiO, J. A.; FURLANI, A. M. C. (Eds.). Recomendações de adubação e calagem para o Estado de São Paulo. Campinas: Instituto Agronômico, 1996. p. 175. (Boletim técnico, 100).

TRANI, P. E.; RAIJ, B. van. Hortaliças. In: RAIJ, B. van; CANTARELlA, H.; QUAGGIO, J. A.; FURLANI, A. M. C. (Eds.). Recomendações de adubação e calagem para o Estado de São Paulo. Campinas: Instituto Agronômico, 1996. p. 157-164. (Boletim técnico, 100).

VIGIER, B.; CUTCLIFFE, J. A. Effect of boron and nitrogen on the incidence of hollow stem in broccoli. Acta Horticulturae, The Hague, v. 157, p. 303-309, 1984. 